\title{
Experiments at LEAR
}

\author{
D.V. Bugg, London
}

(Queen Mary College)

\begin{abstract}
Although CERN is most celebrated for high energy physics, the availability of antiprotons in fluxes unobtainable hitherto has opened a new field of experiments in the $100 \mathrm{MeV}$ range.
\end{abstract}

The main thrust of experiments at CERN these days is with antiprotons. The famous discovery of the $\mathrm{W}$ and the $Z$ particles was made possible by the creation of an intense, high brightness source of $\bar{p}$. A bonus is that $\bar{p}$ are now available for experiments at low energy on meson spectroscopy, exotic atoms and nuclear physics. For this purpose, they are stored in LEAR, which stands for 'Low Energy Antiproton Ring'. Typically, a little over $10^{9} \bar{p}$ are stored in LEAR, and are spilled out slowly and continuously into three beamlines, with an intensity of $10^{5} \bar{p}$ per second in each channel. These beams are an immense improvement on what has previously been available, not only in intensity, but also in beam quality and purity. Previous $\overline{\mathrm{p}}$ beams were contaminated by a huge background of $\pi^{-}, \mu^{-}$and $e^{-}$, but at LEAR this background is completely absent; the $\pi$ and $\mu$ decay away in the storage rings in a few microseconds, and the electrons generate bremsstrahlung and disappear rapidly.

The gymnastics required to achieve this are illustrated in Fig. 1. Every $2 \mathrm{~s}$, protons are accelerated to $26 \mathrm{GeV} / c$ in the PS and are fired into a target to produce $3.5 \mathrm{GeV} / c \overline{\mathrm{p}}$. These $\overline{\mathrm{p}}$ are captured in the antiproton accumulator (AA), stored for anything from 1 hour to many days, and are 'cooled'. The cooling process acts on the principle of Maxwell's demon. Probes at one side of the accumulator measure the beam distribution and momentum spread, and feed signals to the other side of the ring, where accelerating cavities apply Fourier components with frequency and phase appropriate to reduce the size and momentum spread of the beam. This process is called 'stochastic cooling'. About once an hour, after the beam in the $A A$ has shrunk to a small phase space $\Delta p \Delta x$ with momentum spread $\Delta p / p \leq 0.003$, part of the beam is peeled off through transfer line TTL2 and sent back to the PS. There, normal operation is temporarily interrupted in order to decelerate the beam to $610 \mathrm{MeV} / \mathrm{c}$, when it is finally transferred to LEAR. In the deceleration process, $\Delta p \Delta x$ remains constant (Liouville's theorem), and since $\Delta p$ decreases, $\Delta x$ increases. The stochastic cooling in the AA is essential for the $\bar{p}$ beam to fit into the aperture of the PS and LEAR.

In LEAR itself, the beam can be further decelerated without significant loss to $200 \mathrm{MeV} / \mathrm{c}$ or accelerated to a maximum momentum of $2 \mathrm{GeV} / c$. There will eventually be provision for further cooling in LEAR, to compensate for gradual heating of the beam by noise and Coulomb scattering by the residual atoms in the vacuum of the ring. This will be done by 'electron cooling', a process where the circulating $\bar{p}$ beam runs through an intense ( $1 \mathrm{~A}$ ) electron beam with velocity equal to that of the $\bar{p}$ but smaller phase space; the two beams come to thermal equilibrium via Coulomb scattering, and a $\bar{p}$ beam size of $1 \mathrm{~mm}$ with a momentum spread of 1 part in $10^{5}$ should be achievable. As yet, electron cooling is not available.

The first physics run at LEAR took place for a few hectic days in August 1983 , and was followed by two ten-day runs in November and December. Preliminary results are now coming through, and LEAR is scheduled to run for 61 days in 1984. Fifteen experiments are eager for beam.
As far as particle physics is concerned, the interest in low momentum antiprotons lies in the study of meson resonances. The $\bar{p} p$ channel has the quantum numbers of non-strange mesons with isospin 0 and 1 . The energy available allows direct formation of resonances $X(M)$ with mass $M>1876$ $\mathrm{MeV} / \mathrm{c}^{2}, \overline{\mathrm{p} p} \rightarrow \mathrm{X}$, or indirect formation of lighter mesons via processes such as $\bar{p} p$ $\rightarrow \pi \mathrm{X}$. Present belief is that the lightest mesons $\pi(140), \rho(770), \omega(782)$ and $\phi(1020)$ are made up of a quark $q$ and an anti-quark $\bar{q}$, each carrying spin $\frac{1}{2}$; these mesons are the ${ }^{1} S_{0}$ and ${ }^{3} S_{1}$ states of the q $\bar{q}$ system. Heavier mesons are expected to fill energy levels analogous to those of the hydrogen atom; several are known in the mass range 1000 to 1500 $\mathrm{MeV} / \mathrm{c}^{2}$ and probably correspond to $n=$ 2 states with quantum numbers ${ }^{1} \mathrm{~S}_{0}$, ${ }^{3} \mathrm{~S}_{1},{ }^{3} \mathrm{P}_{0},{ }^{3} \mathrm{P}_{1},{ }^{3} \mathrm{P}_{2}$ and ${ }^{1} \mathrm{P}_{1}$, and even heavier mesons are believed to be $n=3 q \bar{q}$ states.

However, what makes the mass range accessible at LEAR of great interest is the possible existence of resonances of a qualitatively different type. The currently fashionable theory of strong interactions (QCD) implies that quarks interact by exchange of 'coloured gluons', analogous to heavy photons, but carrying an extra quantum number called 'colour'. Although non-perturbative calculations are not yet possible, an expectation is that mesons made up of two (or more) gluons, gg, called glueballs, should exist with masses in the range 1000 to $1700 \mathrm{MeV} / \mathrm{c}^{2}$. Several candidates exist, but the situation is debatable because present data are inadequate to establish complete families of resonances. Other possible states are "hermaphrodites", qव̄g. These could

Fig. 1 - The accelerator complex at CERN. PS is the $26 \mathrm{GeV} / \mathrm{C}$ Proton Synchrotron; ISR the Intersecting Storage Rings; PSB the PS Booster; AA the Antiproton Accumulator. The $2.2 \mathrm{~km}$ diameter ring of the $500 \mathrm{GeV} / \mathrm{c}$ Proton Synchrotron is off the drawing, above the ring of the PS.

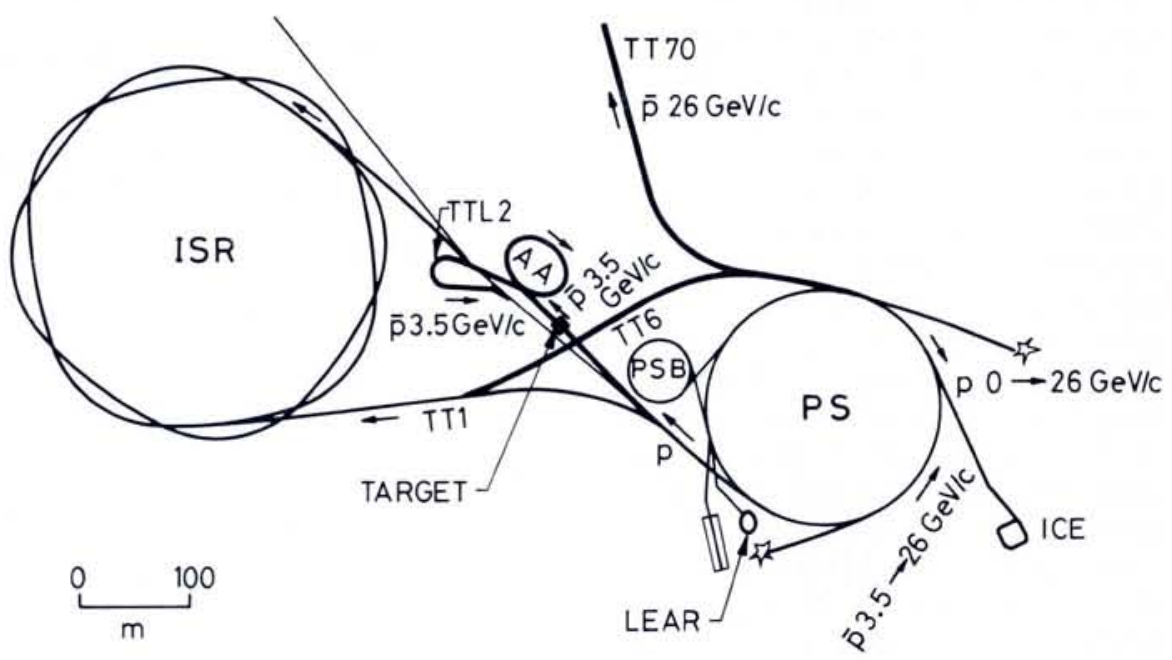



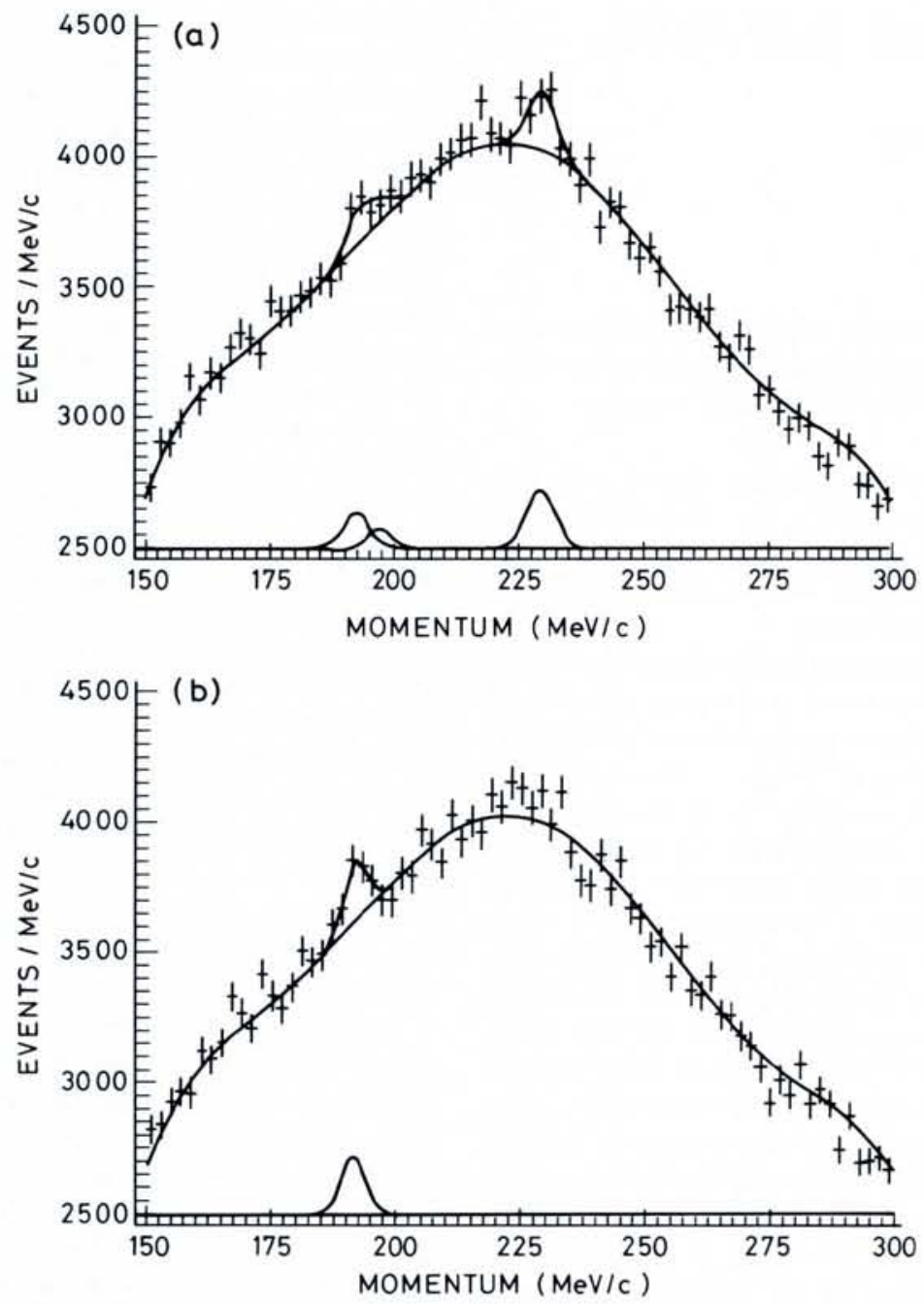

Fig. 2 - The spectra of momenta observed in $\overline{p p}$ annihilation at rest in liquid hydrogen (a) $\pi^{+}$, (b) $\pi^{-}$. exist with quantum numbers (hence decay modes) not accessible to q⿳亠丷厂 or gg states. The most likely situation is that physical mesons are linear combinations these possibilities, comprehensive data on all decay modes are essential; branching ratios between decay modes are an important clue to the coefficients $\alpha, \beta$ and $\gamma$.

Over the decade before LEAR started, there were many tentative indications of narrow resonances, one seen in the total cross-section $\overline{\mathrm{p} p} \rightarrow \mathrm{X}$, and several others in $\bar{p} p \rightarrow \gamma \mathrm{X}$ or more complicated processes. Several early experiments at LEAR have sought, without success, to confirm these narrow resonances. The Basel-Stockholm-Thessaloniki collaboration has measured the $\gamma$ spectrum from $\bar{p} \mathrm{p}$ interactions at rest. Preliminary results reported in January show no sign of three narrow $\gamma$ peaks reported earlier (with poor statistics and huge background). The Amsterdam-Geneva-OMCSurrey-Trieste collaboration has measured $\bar{p}$ p total cross-sections from 385 to $600 \mathrm{MeV} / \mathrm{c}$ with considerably better sta$\alpha q \bar{q}+\beta q \bar{q} g+\gamma g g$. In order to untangle tistics and resolution than earlier measurements; results are very smooth with momentum showing no sign of a peak previously claimed as $\bar{p} p \rightarrow S(1939)$ at $500 \mathrm{MeV} / \mathrm{c}$. In one way, these negative results are disappointing, since several experiments were equipped to follow up positive results. On the other hand, it would have been hard to understand the existence of many narrow states, since the energy available should make decays so rapid that resonance widths of the order $100-200 \mathrm{MeV}$ are to be expected.

One positive result from the AthensIrvine-New Mexico-Pennsylvania StateTemple collaboration is shown in Fig. 2. They have measured $\pi^{ \pm}$momentum spectra in $\bar{p}$ p annihilations at rest in liquid hydrogen, in a search for $\bar{p} p$ $\rightarrow \pi^{ \pm} X^{\mp}$. In the $\pi^{+}$spectrum, Fig. 2(a), there are narrow peaks (compatible with experimental resolution) at 230 and 192 $\mathrm{MeV} / c$, while in the $\pi^{-}$spectrum, Fig. 2 (b), only the latter is apparent. The peak at $230 \mathrm{MeV} / \mathrm{c}$ has an innocent explanation. Roughly $4 \%$ of annihilations produce $\mathrm{K}^{+}$and $\mathrm{K}^{-}$. If the $\mathrm{K}^{+}$stop in the target liquid, they have a dominant decay mode $\mathrm{K}^{+} \rightarrow \mu^{+} \nu$ responsible for the observed peak. (Stopping $\mathrm{K}^{-}$give no equivalent peak, since they are captured by protons and undergo the exothermic reactions $\mathrm{K}^{-} \mathrm{p} \rightarrow \Lambda^{\circ} \pi^{\circ}$ or $\Sigma \pi$.) A complication is that another $\mathrm{K}^{+}$decay mode, $\mathrm{K}^{+} \rightarrow \pi^{+} \pi^{\circ}$ produces a peak at $196 \mathrm{MeV} / \mathrm{c}$, of calculable height, shown at the bottom of Fig. 2(a). The surviving peaks at $192 \mathrm{MeV} / \mathrm{c}$, of equal height in $\pi^{+}$and $\pi^{-}$, as they should be, are taken as evidence for $\bar{p} p \rightarrow C^{ \pm}(1620) \pi^{\mp}$. The letter $\mathrm{C}$ stands for Cordelia, the only one of the three daughters of King Lear who loved him and did not tell lies. Let's hope the name is appropriate! Confirmation with improved resolution (which would improve signal/background) is desirable, since the Asterix group, who have studied $\bar{p} p$ annihilations at rest in hydrogen gas, see no evidence for this peak. However, as we shall see below, there are physics reasons why annihilation could be different in liquid and gas.

Further progress on broad meson resonances is likely to require phase sensitive detection, available in polarisation measurements. Two groups are studying this in $\bar{p} p$ annihilation into two body
Fig. 3 - Energy levels of the $\overline{p p}$ system.

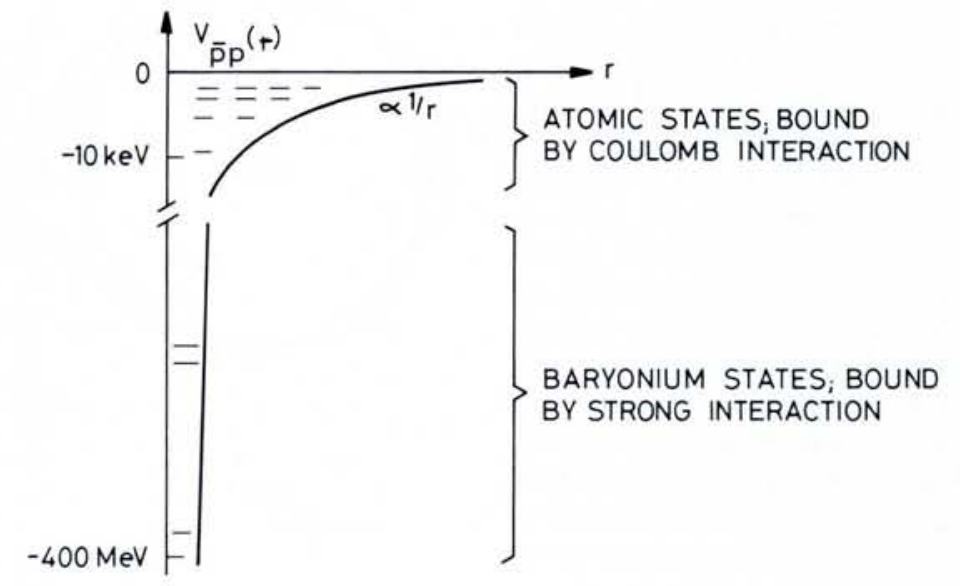




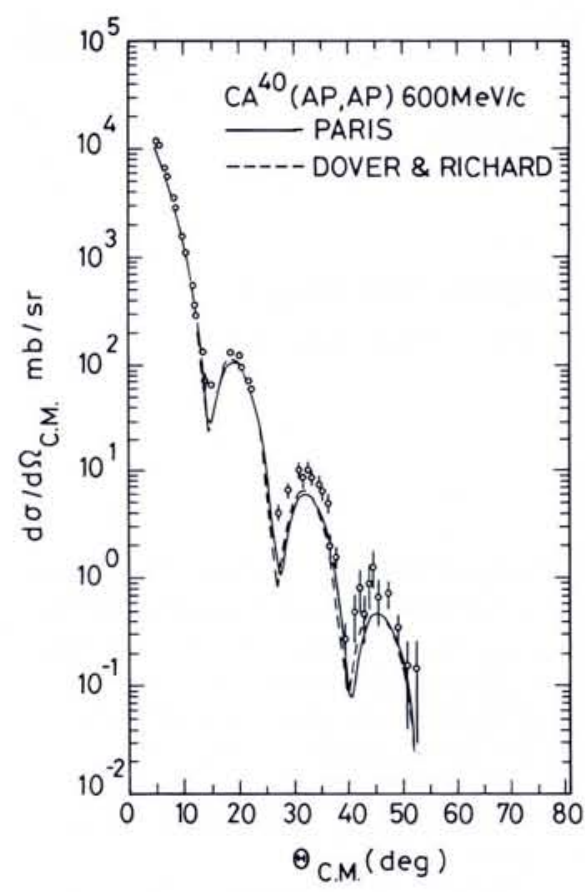

Fig. 4 - The angular distribution of $\bar{p}$ elastic scattering from $\mathrm{Ca}^{40}$ at $600 \mathrm{MeV} / \mathrm{c}$.

channels: $\pi^{-} \pi^{+}, K^{-} K^{+}, \bar{p} p, \Lambda^{\circ} \bar{\Lambda}^{\circ}$ and $\Sigma^{\circ} \Sigma^{\circ}$. The $\Lambda^{\circ} \bar{\Lambda}^{\circ}$ channel is interesting since $\Lambda^{\circ}$ and $\bar{\Lambda}^{\circ}$ decays give rise to a well known asymmetry which measures the polarisation of the parent; in this channel, one can therefore measure, in addition, spin correlations between $\Lambda^{\circ}$ and $\bar{\Lambda}^{\circ}$.

There are two other experiments producing data on meson resonances. The Asterix collaboration (CERN-OrsayMainz-Munich-TRIUMF-Zurich) has a powerful $4 \pi$ detector for charged particles from $\bar{p} p$ annihilations, together with limited $\gamma$ detection. They have already accumulated more than 10 times the previous bubble chamber statistics on $\overline{\mathrm{p} p}$ annihilation at rest. Secondly, a Torino-Manchester-Saclay-Padova-Annecy collaboration is studying the electromagnetic form factor of the nucleon in the rare process $\overline{\mathrm{p}} \mathrm{p} \rightarrow \mathrm{e}^{-} \mathrm{e}^{+}$(branching ratio $10^{-8}$ ); they also trigger on $\bar{p} p$ $\rightarrow \mathrm{e}^{+} \mathrm{e}^{-} \mathrm{X}$ and expect to be sensitive to vector mesons $\left(J^{P}=1^{-}\right)$.

Let us now look more closely at the atomic physics involved when a $\bar{p}$ stops and is captured by an atom or molecule. Initially, the $\bar{p}$ is captured into a very highly excited state by a hydrogen molecule, winkling out one electron. At such high excitations, the molecule de-excites preferentially by the Auger process, kicking out the second electron. The result is a neutral $\bar{p} p$ atom, which de-excites by photon emission, populating preferentially those states with high angular momentum $\ell=n$ (Fig. 3). Left to its own devices, the atom would de-excite like this until overlap between $p$ and $\bar{p}$ is sufficient for annihilation to occur. However, the neutral $\bar{p} p$ atom is free to drift through other molecules, and as it does so it feels strong electric fields, which induce Stark mixing. This mixing temporarily induces some $\mathrm{S}$ state into the wave function, and the annihilation probability is greatly enhanced. It is believed that when $\bar{p}$ stop in liquid hydrogen, annihilation takes place almost purely from atomic S states. The Asterix group has found that annihilations in hydrogen gas look rather different from results observed earlier in liquid hydrogen bubble chambers. They conclude that in gas the annihilation takes place dominantly in $\mathrm{P}$ states.

In the $1 \mathrm{~S}$ state, the radius of the Bohr orbit is so small that the energy level is significantly shifted by the nuclear potential. It would be interesting to observe this shift by measuring the energies of $\mathrm{X}$-rays emitted in transitions to the ground state. The shift measures the real part of the $\bar{p} p$ interaction at rest; the width measures the imaginary part. Three groups have attempted to observe $\mathrm{L}$ and $\mathrm{K} X$-rays. Technically this is tricky, since they have energies of respectively $2 \mathrm{keV}$ and 9-12 keV, hence poor transmission through materials. None of the groups has yet seen $\mathrm{K} X$-rays, and upper limits have been set of about $10^{-3}$ for the yield of $\mathrm{K} X$-rays per $\overline{\mathrm{p}}$ stopping. The Karlsruhe group is working at hydrogen pressures below 1 atmosphere, in order to reduce Stark mixing. The $\mathrm{L} \mathrm{X}$-rays have been observed, confirming strong $P$ wave annihilation.

With heavier nuclei, the atomic physics is similar, except that Stark mixing is absent. The interest lies in following the $\mathrm{X}$-ray spectrum to the radius at which annihilation takes over. This explores sensitively the extreme surface of the nucleus, as well as the range of the $\bar{p} \mathrm{~N}$ interaction. By working with separated isotopes, e.g. $\mathrm{O}^{16}$ and $\mathrm{O}^{18}$, where the shell model predicts precise wave functions for the last two valence neutrons in $\mathrm{O}^{18}$, one can explore the $\overline{\mathrm{p}} \mathrm{n}$ interaction separately from the $\bar{p} p$ interaction. From those $\mathrm{X}$-rays where nuclear interactions are negligible, one gets a precise measurement of the $\bar{p}$ mass $m$ and magnetic moment $\mu$. A very fundamental theorem (the CPT theorem) predicts that $m(\overline{\mathrm{p}})=$ $m(\mathrm{p})$ and $\mu(\overline{\mathrm{p}})=-\mu(\mathrm{p})$. Any departure from exact equality would be of fundamental significance.

The $\bar{p}$-nucleus interaction can also be studied by scattering $\bar{p}$ from nuclei. The earliest LEAR results were obtained by Garreta et al., using a high resolution spectrometer to study $\bar{p}$ elastic scattering from $\mathrm{C}^{12}, \mathrm{Ca}^{40}$ and $\mathrm{Pb}^{208}$. Strong dif- fraction patterns are observed (Fig. 4), confirming that the $\bar{p}$-nucleus interaction is strongly absorptive, and eliminating an unlikely fit to earlier X-ray spectra in which the $\bar{p}$-nucleus interaction was dominantly real.

Up to now, LEAR operation has been only at 300 and $600 \mathrm{MeV} / \mathrm{c}$, but during 1984 , running at $1500 \mathrm{MeV} / \mathrm{c}$ is also forseen. The tuning of the extraction system is delicate and slow, and has hindered running other momenta. One looks forward eagerly to the availability of the full range of momenta, to allow exploration in detail of the rich spectrum of meson resonances, particularly the striking glueball candidates observed elsewhere with masses from 2200 to 2400 $\mathrm{MeV} / \mathrm{c}^{2}$.

\section{University of Bristol}

\section{H.H. Wills Physics Laboratory}

Applications are invited for the post of Research Associate in Theoretical Physics, tenable for three years beginning as soon as possible. Preference will be given to applicants sharing research interests with members of the Theoretical Physics Group at the University of Bristol; these interests include condensed theory (R. Evans, D.A. Greenwood and B.L. Gyorffy) elementary particle theory (J.W. Alcock, W.N. Cottingham and B.R. Pollard) and nonlinear physics (M.V. Berry and J.H. Hannay).

Applications should be sent to The Registrar and Secretary, Senate House, Tyndall Avenue, Bristol BS8 1TH by 15 September 1984. 